\title{
Middle Meningeal Artery Embolization for the Treatment of Bilateral Chronic Subdural Hematoma
}

\author{
Qi Wei ${ }^{1 \dagger}$, Gangxian Fan ${ }^{1,2+}$, Zhenzhu $\mathrm{Li}^{1,3,4}$, Qingbo Wang ${ }^{1}, \mathrm{Ke} \mathrm{Li}^{1}$, Chao Wang ${ }^{1 *}$ and \\ Zefu $\mathrm{Li}^{1 *}$ \\ ${ }^{1}$ Department of Neurosurgery, Binzhou Medical University Hospital, Binzhou, China, ${ }^{2}$ Department of Neurosurgery, Linyi \\ People's Hospital, Linyi, China, ${ }^{3}$ Department of Neursosurgery, Hwa Mei Hospital, University of Chinese Academy of \\ Sciences, Ningbo, China, ${ }^{4}$ Ningbo Institute of Life and Health Industry, University of Chinese Academy of Sciences, Ningbo, \\ China
}

OPEN ACCESS

Edited by:

Osama O. Zaidat,

Northeast Ohio Medical University,

United States

Reviewed by:

Luis Rafael Moscote-Salazar, University of Cartagena, Colombia

Qazi Zeeshan,

University at Buffalo, United States

${ }^{*}$ Correspondence:

Chao Wang

chaowang_0628@163.com

Zefu Li

lizefu163@163.com

†These authors have contributed equally to this work and share first

authorship

Specialty section

This article was submitted to Endovascular and Interventional

Neurology,

a section of the journal

Frontiers in Neurology

Received: 08 March 2021 Accepted: 21 September 2021

Published: 28 October 2021

Citation:

Wei Q, Fan G, Li Z, Wang Q, Li K,

Wang $C$ and Li Z (2021) Middle Meningeal Artery Embolization for the

Treatment of Bilateral Chronic

Subdural Hematoma.

Front. Neurol. 12:651362.

doi: 10.3389/fneur.2021.651362
Background: Bilateral chronic subdural hematoma (bCSDH) is a frequent condition commonly linked to the need for retreatment; however, the reason for this high retreatment rate remains unclear. The middle meningeal artery (MMA) was found to have a relationship with the occurrence and development of chronic subdural hematomas. This study examines a possible method to reduce bCSDH recurrence using bilateral MMA embolization combined with bilateral burr-hole drainage.

Materials and Methods: Ten patients with bCSDH who underwent bilateral MMA embolization combined with bilateral burr-hole drainage at our hospital between June 2018 and May 2020, were retrospectively analyzed. Patients' clinical information, prognoses, imaging results, as well as surgical results were documented and analyzed.

Results: Ten patients were diagnosed with bCSDH with no comorbid brain diseases. They underwent bilateral MMA embolization combined with bilateral burr-hole drainage. We embolized the MMA immediately before burr hole drainage successfully and employed angiography to validate these results. All the patients attained relief of symptoms without adverse events, and no re-expansion or relapse was reported in the follow-up computed tomography.

Conclusion: Bilateral MMA embolization combined with bilateral burr-hole drainage is an available treatment for patients with bCSDH and may have the potential for preventing recurrence.

Keywords: hematoma, subdural, chronic, meningeal arteries, interventional embolization, neurosurgery

\section{INTRODUCTION}

Chronic subdural hematoma (CSDH) is among the most frequent conditions, with incidence rates varying from 5.3 to 13.5 cases per 100,000 person years in the general population and with a higher incidence in the older population (1). Generally, CSDH is regarded a delayed sequela of head trauma, resulting in the infiltration of phagocytic macrophages and neovascular proliferation at the site of hematomas (2). CSDH is encountered frequently in neurology clinics. Unilateral CSDH 
( $\mathrm{uCSDH}$ ) occurs in a remarkable number of individuals with $\mathrm{CSDH}$, however bilateral $\mathrm{CSDH}(\mathrm{bCSDH})$ is occasionally encountered in neurosurgical practice. Cases of bCSDH, which account for about $14-25 \%$ of all $\mathrm{CSDH}$, are believed to be a distinct subgroup from uCSDH (3-5). Presently, surgical evacuation with craniotomy or burr holes constitutes the main treatment of symptomatic $\mathrm{CSDH}(6,7)$. In researches on CSDH treatment with burr-hole surgery, $11-33 \%$ of operative cases experienced a relapsed hematoma (8-10). Initially, most clinicians considered bCSDH as equivalent to $\mathrm{uCSDH}$ since there was no distinct difference in either disease presentation or treatment strategy. Non-etheless, research evidence has documented that bCSDH cases were linked to increased retreatment rates $(4,11)$. Others have also documented that bCSDH was an independent predictor of CSDH relapse although the cause of this elevated rate of relapse remains unclear $(11,12)$.

The middle meningeal artery (MMA) may play an indispensable role in $\mathrm{CSDH}$ onset and progress. As reported in the literature, there was a rich blood vessel network on the dura mater, and the blood supply of the dura mater at the fronto-tempo-parietal area was mainly derived from the branch of the external carotid artery-the MMA (13-16). Histological investigations have illustrated that some small blood vessels from the MMA were found to be connected to the CSDH outer membrane through the dura mater $(6,17-20)$. Therefore, embolization of MMA might be a beneficial strategy for treating refractory or recurrent CSDH. Recently, MMA embolization has been described as an alternative treatment for relapsed or primary CSDH. Studies on performing MMA embolization for treating individuals with $\mathrm{CSDH}$ have also been published $(17,21,22)$. Embolization of the MMA was found to be efficient for individuals with refractory CSDH or CSDH with relapse risk, and was reported as a beneficial treatment approach to avert enlargement of hematoma (23).

Despite some researches reporting the use of MMA embolization for treating individuals with $\mathrm{CSDH}$, none, to our knowledge, have documented on the effect of bilateral MMA embolization as a pre-treatment strategy for bCSDH to prevent the associated high recurrence rate. Herein, we document on the effective utility of bilateral MMA embolization combined with bilateral burr-hole drainage to treat patients with bCSDH.

\section{MATERIALS AND METHODS}

\section{Patient Selection}

Data for 10 individuals with bCSDH treated at our center from June 2018 to May 2020 were analyzed retrospectively. The inclusion criteria were: (A) all patients clearly diagnosed with bCSDH via brain computed tomography (CT); (B) patients with symptomatic bCSDH; and (C) >14 days must have elapsed from the time of head trauma to the time of performed operation. The exclusion criteria consisted of: (a) $\mathrm{CSDH}$ cases with comorbid conditions (vascular lesions, brain tumor, arachnoid cyst, spontaneous intracranial hypotension, or previous craniotomy); (b) poor medical condition with life expectancy of $<6$ months. The study approval was granted by the ethics committee of Binzhou Medical University Hospital (No. 2018065). All individuals analyzed in this study provided informed consent. The study protocol adhered to the Declaration of Helsinki.

\section{Clinical Evaluation}

The clinical data of 10 patients were documented, including their basic information such as age and gender, clinical manifestation, operation method and time, and follow-up time. The GCS (Glasgow Coma Scale) was employed to determine the preoperative neurological function. The collected data were checked, unified, and summarized into a table form.

\section{MMA Embolization}

A local anesthetic was administered prior to bilateral MMA embolization. Afterwards, Seldinger's technique was employed to puncture the femoral artery. A standard 6-French sheath was utilized to gain entry into the femoral artery. The guiding catheter was placed in the external carotid artery. Next, angiography of the external carotid artery was conducted, in which an Excelsior ${ }^{\circledR}$ SL-10 microcatheter (Target Therapeutics/Boston Scientific, Fremont, California, USA) was placed under the guidance of fluoroscopy and the Traxcess ${ }^{\circledR} 14$ microguidewire (MicroVention, California, USA) was placed into the trunk both middle meningeal arteries respectively $(10,23)$. Under blank fluoroscopic roadmap control, embolization was performed by coiling to the embolization site through the microcatheter. Several types of coils were used: Detachable coils (Target ${ }^{\circledR}$ detachable coil, Boston Scientific, California, USA), Microplex (Microvention, California, USA), and Axium (ev3, California, USA). Next, we confirmed these results by intraoperative angiography, the guiding catheter along with the femoral arterial sheath were removed, followed by placing of the occlusion in the puncture site of the femoral artery. The all patients was treated using MMA embolization before burr-hole drainage to mitigate the symptoms, promote prognosis, as well as decrease the chances of relapse. After the operation, we moved all the study participants into the ward for observation.

\section{Follow-Up}

On the admission of each participant, a cranial CT scan was employed to diagnose hematomas, with the imaging conducted once every week or as per the changes in clinical symptoms as needed by each individual patient during hospitalization. Incidences of post-surgery acute intra-cranial bleeding after treatment of bCSDH using surgery were documented. Upon improvement of the symptoms, all the study subjects were discharged. A brain CT once in a month was recommended and a 3-4 month follow-up was performed for all the participants. The GOS (Glasgow Outcome Scale) along with mRS (modified Rankin Scale) were employed to evaluate the post-surgery neurological function at the final clinic visit. When the symptoms worsened or reappeared, the involved patient was re-admitted. Recurrence of CSDH was defined as patients who had worsening symptoms as illustrated by the CT-verified re-accumulation of $\mathrm{CSDH}$ at- or near an initial hematoma site post the initial surgery, as well as those who needed a repeat surgery (3). 
TABLE 1 | Baseline characteristics and prognoses of patients with bilateral chronic subdural hematoma.

\begin{tabular}{|c|c|c|}
\hline \multicolumn{2}{|c|}{ Characteristics } & \multirow{2}{*}{$\begin{array}{c}\text { No. of patients } \boldsymbol{N}=10 \\
2\end{array}$} \\
\hline Age (year) & $40 \leq,<60$ & \\
\hline & $\geq 60$ & 8 \\
\hline \multirow[t]{3}{*}{ Hematoma location } & ${ }^{a} b-P a r i e t a l$ & 2 \\
\hline & b-Fronto-parietal & 2 \\
\hline & b-Fronto-parieto-temporal & 6 \\
\hline \multirow[t]{5}{*}{ Clinical symptoms } & Headache & 6 \\
\hline & Nausea, vomiting & 3 \\
\hline & Slurred speech & 1 \\
\hline & Unsteady gait & 2 \\
\hline & Weakness in both lower limbs & 2 \\
\hline \multirow[t]{2}{*}{ bDrug therapy } & Yes & 1 \\
\hline & No & 9 \\
\hline \multirow[t]{2}{*}{${ }^{\circ} \mathrm{GCS}$} & 14 & 2 \\
\hline & 15 & 8 \\
\hline \multirow[t]{2}{*}{${ }^{\mathrm{d}} \mathrm{GOS}$} & O-IV & 0 \\
\hline & V & 10 \\
\hline \multirow[t]{2}{*}{${ }^{e} \mathrm{mRS}$} & 0 & 6 \\
\hline & 1 & 4 \\
\hline
\end{tabular}

${ }^{a}$ b, bilateral; ${ }^{b}$ Drug therapy, current anticoagulant or antiplatelet therapy; ${ }^{c} \mathrm{GCS}$, Glasgow Coma Scale; ${ }^{d}$ GOS, Glasgow Outcome Scale; ${ }^{e} \mathrm{mRS}$, modified Rankin Scale.

\section{RESULTS}

A summary of the baseline features of the study participants is provided in Table 1. All the 10 study subjects were men (mean age $=63.1$ years; range $40-76$ years). All the 10 study subjects had a head trauma history, and the GCS was higher than 13 on the admission date for all participants. The most common symptoms among the subjects included headache, nausea, vomiting, limb weakness, unsteady gait, and slurred speech. The imaging data of all the participants were verified prior to the operation. A summary of the surgical methods and time of the study subjects, and the follow-up time is provided in Table 2. All the study subjects were treated with bilateral MMA embolization combined with bilateral burr-hole drainage. Embolization was successful in all the participants, with no adverse event reported, and bleeding was promptly stopped. No rebleeding occurred during the 3-4 month follow-up as evidenced by the brain CT scans. Moreover, the size of the hematoma cavity decreased gradually, thereafter.

\section{Representative Cases' Clinical Data Patient 1}

Patient 1 was treated for coronary heart disease with oral aspirin. He had minor head trauma history 25 days before and visited a neighborhood hospital with a complaint of headache. He underwent conservative treatment and regular follow-ups were requested. The patient was not required to stop aspirin medication owing to the high risks associated with its discontinuation. Head CT performed 25 days after trauma showed a slight enlargement of the bilateral hematoma and increased density (Figure 1A). Meanwhile, his symptoms became
TABLE 2 | The patient's operation method, operation time and follow-up time.

\begin{tabular}{|c|c|c|c|c|}
\hline Patient & $\begin{array}{l}\text { Operation } \\
\text { method }\end{array}$ & $\begin{array}{c}\text { Time of } \\
\text { MMA }^{\mathrm{a}} \\
\text { embolization } \\
\text { (min) }\end{array}$ & $\begin{array}{l}\text { Time of burr } \\
\text { hole } \\
\text { dranage } \\
\text { (min) }\end{array}$ & $\begin{array}{l}\text { Follow-up } \\
\text { time (days) }\end{array}$ \\
\hline 1 & $\begin{array}{l}\text { MMA embo } \\
\text { and burr holes }\end{array}$ & 55 & 60 & 106 \\
\hline 2 & $\begin{array}{l}\text { MMA embo } \\
\text { and burr holes }\end{array}$ & 48 & 63 & 97 \\
\hline 3 & $\begin{array}{l}\text { MMA embo } \\
\text { and burr holes }\end{array}$ & 60 & 72 & 122 \\
\hline 4 & $\begin{array}{l}\text { MMA embo } \\
\text { and burr holes }\end{array}$ & 52 & 65 & 115 \\
\hline 5 & $\begin{array}{l}\text { MMA embo } \\
\text { and burr holes }\end{array}$ & 50 & 70 & 120 \\
\hline 6 & $\begin{array}{l}\text { MMA embo } \\
\text { and burr holes }\end{array}$ & 45 & 68 & 98 \\
\hline 7 & $\begin{array}{l}\text { MMA embo } \\
\text { and burr holes }\end{array}$ & 58 & 75 & 115 \\
\hline 8 & $\begin{array}{l}\text { MMA embo } \\
\text { and burr holes }\end{array}$ & 54 & 64 & 130 \\
\hline 9 & $\begin{array}{l}\text { MMA embo } \\
\text { and burr holes }\end{array}$ & 55 & 68 & 108 \\
\hline 10 & $\begin{array}{l}\text { MMA embo } \\
\text { and burr holes }\end{array}$ & 52 & 66 & 112 \\
\hline
\end{tabular}

${ }^{a} M M A$, middle meningeal artery; ${ }^{b} M M A$ embo and burr holes: bilateral MMA embolization in combination with bilateral burr hole drainage.

more serious as the headache worsened, and he developed a new symptom of vomiting. These results were regarded as aggravation signs. Thus, bilateral MMA embolization combined with bilateral burr-hole drainage was advised and performed under local anesthesia in our hospital. Cerebral angiography was conducted pre-, as well as post-surgery for MMA embolization (Figures 1E-H). As proof of proper embolization, the MMA could not be observed during post-surgery angiography. His symptoms improved postoperatively. A repeat brain CT scan exhibited no re-bleeding prior to discharge, and the hematoma cavity gradually decreased in size (Figures 1B-D). No recurrence was observed from discharge to 3-4 months after discharge.

\section{Patient 2}

Patient 2 was admitted to a neighborhood hospital after experiencing a fall, hence received a hard blow to the back of the head. Within 20 days, his follow-up head CT scan revealed thin bCSDH. The patient received conservative treatment and was followed up. Since bilateral hematoma enlargement was observed 20 days later (Figure 2A), and the patient had slurred speech, he visited our hospital. MMA embolization in combination with bilateral burr-hole drainage was suggested under local anesthesia. Cerebral angiography was carried out pre-, as well as post-surgery for MMA embolization (Figures 2E-H). The MMA could not be observed during postoperative angiography. The symptoms of the patient improved postsurgery. Likewise, a repeat CT scan of the brain revealed no re-bleeding prior to discharge. In addition, the hematoma cavity size decreased gradually, thereafter (Figures 2B-D). 

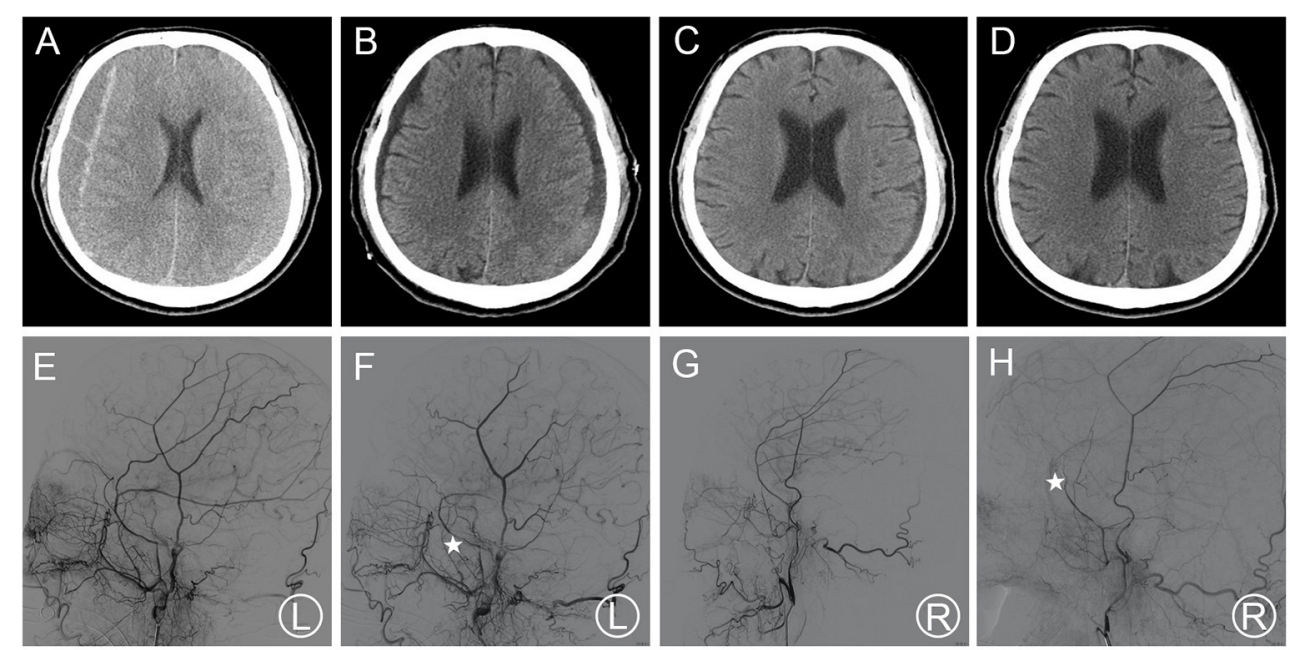

FIGURE 1 | (A) Brain CT images of a hematoma in patient 1 before treatment. (B) Brain CT was repeated before the patient's discharge. (C,D) Follow-up brain CT was performed at 35 and 115 days. (E,F) Pre- and post-operative DSA presented left MMA and the left MMA disappeared (asterisk). (G,H) Pre- and post-operative DSA presented right MMA and the right MMA disappeared (asterisk). DSA, digital subtraction angiography; CT, computed tomography; MMA, middle meningeal artery.
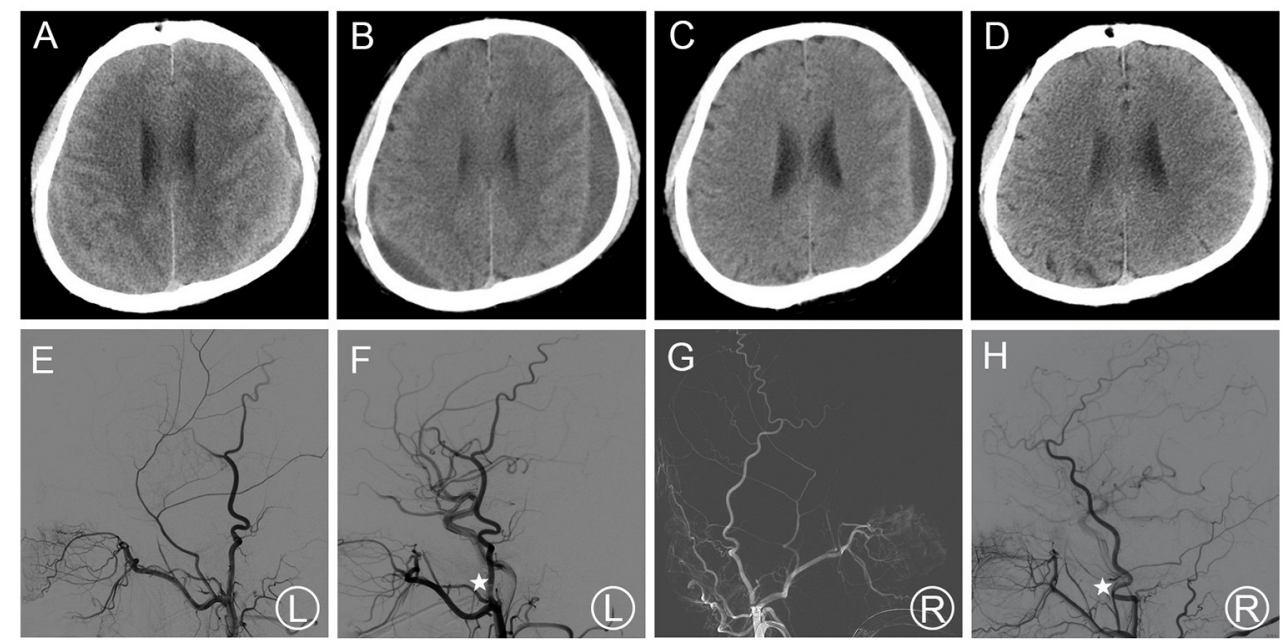

FIGURE 2 | (A) Brain CT images of a hematoma in patient 2 before treatment. (B,C) Brain CT was repeated at 14 days after surgery and before the patient's discharge. (D) Follow-up brain CT was performed at 97 days. (E,F) Pre- and post-operative DSA presented left MMA and the left MMA disappeared (asterisk). (G,H) Pre- and post-operative DSA presented right MMA and the right MMA disappeared (asterisk). DSA, digital subtraction angiography; CT, computed tomography; MMA, middle meningeal artery.

No recurrence was observed from discharge to 3-4 months after discharge.

\section{DISCUSSION}

CSDH is among the most frequent diseases. Its initiation and progress involves complex processes. During this process, the histology of the outer membrane has been the focus of investigation on the mechanisms by which CSDH develops (24). Investigations have showed that the neovascular blood supply of the CSDH outer membrane was mainly MMA $(25,26)$. These findings suggested the possibility of treating CSDH through MMA embolization. Herein, we prevented bCSDH recurrence by embolizing the MMA bilaterally, and no hematoma recurrence was found during the follow-up period after embolization.

Currently, bCSDH is thought to be a distinct subgroup from uCSDH (27). The clinical presentation of bCSDH shows greater diversity than that of uCSDH. Tsai et al. (28), comparing the clinical manifestations in unilateral vs. bilateral $\mathrm{CSDH}$, documented that individuals with bCSDH had a high chance of exhibiting symptoms linked to elevated intracranial pressure, consisting of headache, nausea, vomiting and brain 
herniation, while individuals with uCSDH highly likely exhibited symptoms linked to brain shift, consisting of hemiparesis, which might be linked to the loss of counterbalance related with a unilateral hematoma. Additionally, bCSDH sometimes may be asymptomatic, resulting in a delayed or missed diagnosis because of less specific clinical presentation. Furthermore, bCSDH generally has a poorer clinical outcome and progresses more rapidly than $\mathrm{uCSDH}$. Although a previous research found no significant differences in postoperative outcomes between the bilateral and unilateral CSDH groups (4), in more recent studies, outcomes were poorer in the bCSDH group (3, 5, 29, 30), with bilateral hematoma cited as having an elevated risk of acute aggravation of clinical manifestation due to downward herniation. Therefore, early and rapid surgical drainages of bilateral hematomas are needed to achieve better outcomes and recovery of neurological function and may be performed with success rates similar to those in unilateral hematomas $(5,31,32)$.

Patients with CSDH generally have a good prognosis; however, the postoperative recurrence rate ranges from 11 to $33 \%$ (8). Investigations on recurrence factors have always been a hot spot in CSDH research. Several predictors of CSDH recurrence have been documented consisting of advanced age, arachnoid cyst, alcohol abuse, persistent midline shift, the utilization of perioperative antiplatelet/anticoagulant treatment, bleeding tendency, presence of thick membranes, bilateral $\mathrm{CSDH}$, brain atrophy, lower GCS scores, hematoma density, inflammation markers, poor post-surgery brain re-expansion, post-surgery subdural air aggregation, as well as some technical surgery aspects (9, 12, 28, 33-36). However, some results have not yet been confirmed, and some of these predictors appear to play different roles in bCSDH and uCSDH development. Studies have indicated that the mean age of bCSDH patients was significantly greater than that of uCSDH patients. Old age is a known predictor of $\mathrm{CSDH}$ development and an age of more than 75 years has been documented to be an independent predictor of bCSDH development $(3,37)$. This might be linked to brain atrophy along with increased venous fragility. Although persistent brain shifting was described as the current mainstream theory of recurrence, leading to tearing of the bridging veins because of a lack of surrounding reinforcement, the pathogenesis of recurrent $\mathrm{CSDH}$ cannot be explained satisfactorily by a single theory $(1,11,31)$. These findings may help identify patients at a high risk of CSDH recurrence and assist in developing personalized treatment options to reduce recurrence.

Presently, there is no uniform standard for treating bCSDH, and it has a higher recurrence rate than $\mathrm{uCSDH}$ regardless of whether it was managed via unilateral or bilateral surgery. One study found unilateral surgery for bCSDH to be an independent risk factor for retreatment (1). This study reported that the retreatment rate for individuals under bilateral surgery treatment was $14.1 \%$ (18 of 128 cases), which was remarkably lower relative to the retreatment rate in individuals under unilateral surgery treatment $(28.7 \% ; \quad P<0.004)$. Although unilateral surgery is still very commonly performed for bCSDH, especially when the contralateral hematoma is asymptomatic and thin, after a certain period, the contralateral hematoma might enlarge, causing symptoms, requiring another burr-hole surgery $(30,38)$. Our previous study reported that bCSDH worsened on the contralateral side after unilateral surgery in two patients (39). This phenomenon may be explained by the decrease in the intracranial pressure owing to the unilateral evacuation, permitting expansion of the nonoperated contralateral hematoma. Unilateral drainage could also result in the displacement of the brain toward the operated side, expanding the contralateral subdural space $(1,5,30)$. Furthermore, Lin et al. (31) found that the incidence of the late phase of brain shifting post-evacuation was remarkably greater in bCSDH in contrast with uCSDH, which they proposed might be one factor responsible for the higher symptomatic recurrence rate in patients with bCSDH.

To reduce the recurrence rate of bilateral hematomas, bCSDH should be differentiated from unilateral cases to establish an appropriate surgical strategy. Presently, some studies have reported on small vessel connections between the MMA and outer membranes of $\mathrm{CSDH}$, leading to a new approach to interventional therapy for $\mathrm{CSDH}$, which is MMA embolization (17, 20, 40). Link et al. (6) introduced a novel approach to MMA embolization, which provides a minimally invasive and low-risk initial treatment alternative to surgery for patients with symptomatic CSDH when clinically appropriate. In our previous study (41), we reported on the use of absolute alcohol as an embolic agent to prevent the re-expansion and relapse of acute epidural hematoma and $\mathrm{CSDH}$ via MMA embolization; the hematomas of all patients gradually decreased and disappeared after the procedure, with no instances of reexpansion nor recurrence of the hematoma during follow-up. Although studies have shown that the use of any embolic material (liquid or solid) could achieve the desired effect for the occlusion of the MMA, liquid embolic agents needed to pay attention to whether there is anastomotic branches between the internal and external carotid arteries to prevent the liquid embolic agent flowed into the brain and caused inappropriate embolism $(6,21)$. Therefore, the solid embolic material was safer without excluding this dangerous anastomosis. In our study, 10 patients with bCSDH were treated using bilateral prophylactic embolization of the MMA combined with bilateral burr-hole drainage. Embolization was performed to reduce intraoperative bleeding and postoperative hematoma recurrence. Surgical drainage was performed to alleviate symptoms. For all study subjects, symptoms resolved postoperatively, and they were discharged after showing symptomatic improvement. Follow-up brain CT was performed, and no patients experienced hematoma recurrence for at least 3 months after discharge.

There are several limitations to our study. First, this was a retrospective study with a relatively small sample size and without a control group. Second, data were collected through medical records and imaging reviews and were thus less accurate compared to a hypothetical prospective study. Third, the evaluation period for neurological outcomes varied and followup information was difficult to obtain without scheduled returns to the clinic. Despite these miscellaneous limitations, our study provides useful preliminary information to identify both the clinical features of bCSDH and to assess a new treatment idea to reduce its high recurrence rate. 


\section{CONCLUSIONS}

In summary, although there were similarities between bCSDH and $\mathrm{uCSDH}$ case features, it is known that bCSDH is more likely to recur than uCSDH. Thus, bCSDH treatment requires clinical differentiation from uCSDH to establish an appropriate surgical strategy. Herein, bilateral MMA embolization combined with bilateral burr-hole drainage was an available treatment for patients with bCSDH with the potential for preventing recurrence.

\section{DATA AVAILABILITY STATEMENT}

The original contributions presented in the study are included in the article/supplementary material, further inquiries can be directed to the corresponding authors.

\section{ETHICS STATEMENT}

The study was reviewed and approved by the Ethics Committee of Binzhou Medical University Hospital (No. 2018065). The patients/participants provided their written informed consent to participate in this study.

\section{REFERENCES}

1. Andersen-Ranberg NC, Poulsen FR, Bergholt B, Hundsholt T, Fugleholm K. Bilateral chronic subdural hematoma: unilateral or bilateral drainage? J Neurosurg. (2017) 126:1905-11. doi: 10.3171/2016.4.JNS152642

2. Chihara H, Imamura H, Ogura T, Adachi H, Imai Y, Sakai N. Recurrence of a refractory chronic subdural hematoma after middle meningeal artery embolization that required craniotomy. NMC Case Rep J. (2014) 1:15. doi: 10.2176/nmccrj.2013-0343

3. Hsieh C-T, Su I-C, Hsu S-K, Huang C-T, Lian F-J, Chang C-J. Chronic subdural hematoma: differences between unilateral and bilateral occurrence. $J$ Clin Neurosci. (2016) 34:252-8. doi: 10.1016/j.jocn.2016.09.015

4. Huang Y-H, Yang K-Y, Lee T-C, Liao C-C. Bilateral chronic subdural hematoma: what is the clinical significance? Int J Surg. (2013) 11:5448. doi: 10.1016/j.ijsu.2013.05.007

5. Lee J, Park JH. Clinical characteristics of bilateral versus unilateral chronic subdural hematoma. Korean J Neurotrauma. (2014) 10:4954. doi: 10.13004/kjnt.2014.10.2.49

6. Link TW, Boddu S, Marcus J, Rapoport BI, Lavi E, Knopman J. Middle meningeal artery embolization as treatment for chronic subdural hematoma: a case series. Oper Neurosurg. (2018) 14:556-62. doi: 10.1093/ons/opx154

7. Kotwica Z, Saracen A, Dziuba I. Chronic subdural hematoma (CSH) is still an important clinical problem. Analysis of 700 consecutive patients. Transl Neurosci. (2019) 10:260-3. doi: 10.1515/tnsci-2019-0042

8. Jung Y-G, Jung N-Y, Kim E. Independent predictors for recurrence of chronic subdural hematoma. J Korean Neurosurg Soc. (2015) 57:26670. doi: 10.3340/jkns.2015.57.4.266

9. Amirjamshidi A, Abouzari M, Eftekhar B, Rashidi A, Rezaii J, Esfandiari K, et al. Outcomes and recurrence rates in chronic subdural haematoma. $\mathrm{Br} J$ Neurosurg. (2007) 21:272-5. doi: 10.1080/02688690701272232

10. Link TW, Rapoport BI, Paine SM, Kamel H, Knopman J. Middle meningeal artery embolization for chronic subdural hematoma: Endovascular technique and radiographic findings. Interv Neuroradiol. (2018) 24:45562. doi: $10.1177 / 1591019918769336$

\section{AUTHOR CONTRIBUTIONS}

QW and GF designed and carried out the research, as well as wrote the manuscript. ZeL designed the research, performed report supervision, and revised the article critically for content. $\mathrm{CW}$ designed the research and contributed to the analysis. $\mathrm{ZhL}, \mathrm{QW}$, and $\mathrm{KL}$ participated in the acquisition, analysis, and interpretation of the data and provided clinical advice. All authors contributed to the article and approved the submitted version.

\section{FUNDING}

This research was funded by Binzhou Medical University Scientific Research Foundation (BY2018KJ02 and BY2016KYQD15) along with Shandong Province Natural Science Foundation of China (ZR2018LH007 and ZR2017LH033).

\section{ACKNOWLEDGMENTS}

The authors acknowledge assistance from the endovascular Neurosurgery Research Group members (Department of Neurosurgery, Binzhou Medical University Hospital, Binzhou, Shandong, China).

11. Okano A, Oya S, Fujisawa N, Tsuchiya T, Indo M, Nakamura T, et al. Analysis of risk factors for chronic subdural haematoma recurrence after burr hole surgery: optimal management of patients on antiplatelet therapy. $\mathrm{Br} \mathrm{J}$ Neurosurg. (2014) 28:204-8. doi: 10.3109/02688697.2013.829563

12. Torihashi K, Sadamasa N, Yoshida K, Narumi O, Chin M, Yamagata S. Independent predictors for recurrence of chronic subdural hematoma: a review of 343 consecutive surgical cases. Neurosurgery. (2008) 63:11259. doi: 10.1227/01.NEU.0000335782.60059.17

13. Kerber CW, Newton TH. The macro and microvasculature of the dura mater. Neuroradiology. (1973) 6:175-9. doi: 10.1007/BF00335317

14. Roland J, Bernard C, Bracard S, Czorny A, Floquet J, Race JM, et al. Microvascularization of the intracranial dura mater. Surgical Radiol Anat SRA. (1987) 9:43-9. doi: 10.1007/BF02116853

15. Schachenmayr W, Friede RL. The origin of subdural neomembranes. I. Fine structure of the dura-arachnoid interface in man. The American Pathol. (1978) 92:53-68.13.

16. Friede RL, Schachenmayr W. The origin ofsubdural neomembranes. II Fine structural of neomembranes. Am J Pathol. (1978) 92:69-84.

17. Mino M, Nishimura S, Hori E, Kohama M, Yonezawa S, Midorikawa $\mathrm{H}$, et al. Efficacy of middle meningeal artery embolization in the treatment of refractory chronic subdural hematoma. Surg Neurol Int. (2010) 1:78. doi: 10.4103/2152-7806.73801

18. Edlmann E, Giorgi-Coll S, Whitfield PC, Carpenter KL, Hutchinson PJ. Pathophysiology of chronic subdural haematoma: inflammation, angiogenesis and implications for pharmacotherapy. J Neuroinflammation. (2017) 14:108. doi: 10.1186/s12974-017-0881-y

19. Goodell CL, Mealey JJR. Pathogenesis of chronic subdural hematoma. Exp Stud Arch Neurol. (1963) 8:429-37.

20. Takizawa $K$, Sorimachi $T$, Ishizaka $H$, Osada $T$, Srivatanakul $K$, Momose $\mathrm{H}$, et al. Enlargement of the middle meningeal artery on MR angiography in chronic subdural hematoma. J Neurosurg. (2016) 124:1679-83. doi: 10.3171/2015.5.JNS1567

21. Tempaku A, Yamauchi S, Ikeda H, Tsubota N, Furukawa H, Maeda D, et al. Usefulness of interventional embolization of the middle meningeal artery for 
recurrent chronic subdural hematoma: five cases and a review of the literature. Interv Neuroradiol. (2015) 21:366-71. doi: 10.1177/1591019915583224

22. Kim E. Embolization therapy for refractory hemorrhage in patients with chronic subdural hematomas. World Neurosurg. (2017) 101:5207. doi: $10.1016 /$ j.wneu.2017.02.070

23. Hashimoto $\mathrm{T}$, Ohashi $\mathrm{T}$, Watanabe $\mathrm{D}$, Koyama S, Namatame H, Izawa $\mathrm{H}$, et al. Usefulness of embolization of the middle meningeal artery for refractory chronic subdural hematomas. Surg Neurol Int. (2013) 4:104. doi: 10.4103/2152-7806.116679

24. Cecchini G. Chronic subdural hematoma pathophysiology: a unifying theory for a dynamic process. J Neurosurg Sci. (2017) 61:536-43. doi: 10.23736/S0390-5616.16.03156-8

25. Tsukamoto Y, Oishi M, Shinbo J, Fujii Y. Transarterial embolisation for refractory bilateral chronic subdural hematomas in a case with dentatorubral-pallidoluysian atrophy. Acta Neurochir. (2011) 153:11457. doi: 10.1007/s00701-010-0891-3

26. Rowbotham GF, Little E. New concepts on the aetiology and vascularization of meningiomata; the mechanism of migraine; the chemical processes of the cerebrospinal fluid; and the formation of collections of blood or fluid in the subdural space. Br J Surg. (1965) 52:21-4.

27. Park HS, Park ES, Park JB, Kwon SC, Lyo IU, Kim M-H, et al. Chronic subdural hematomas: comparison between unilateral and bilateral involvement. Korean J Neurotrauma. (2014) 10:55-9. doi: 10.13004/kjnt.2014.10.2.55

28. Tsai T-H, Lieu A-S, Hwang S-L, Huang T-Y, Hwang Y-F, A. comparative study of the patients with bilateral or unilateral chronic subdural hematoma: precipitating factors and postoperative outcomes. J Trauma. (2010) 68:5715. doi: 10.1097/TA.0b013e3181a5f31c

29. Agawa Y, Mineharu Y, Tani S, Adachi H, Imamura H, Sakai N. Bilateral chronic subdural hematoma is associated with rapid progression and poor clinical outcome. Neurol Med Chir. (2016) 56:198-203. doi: 10.2176/nmc.oa.2015-0256

30. Fujitani S, Ishikawa $\mathrm{O}$, Miura $\mathrm{K}$, Takeda $\mathrm{Y}$, Goto $\mathrm{H}$, Maeda $\mathrm{K}$. Factors predicting contralateral hematoma growth after unilateral drainage of bilateral chronic subdural hematoma. J Neurosurg. (2017) 126:7559. doi: 10.3171/2016.1.JNS152655

31. Lin M-S, Chang C-J, Lin C-C, Chen S-T, Hwang B, Lu S-H. Quantitative assessment of brain shifting in the late phase postevacuation in unilateral and bilateral chronic subdural hematomas. Int J Neurosci. (2016) 126:61722. doi: $10.3109 / 00207454.2015 .1051044$

32. Takahashi S, Yamauchi T, Yamamura T, Ogishima T, Arai T. Proposal of treatment strategies for bilateral chronic subdural hematoma based on laterality of treated hematoma. Asian J Neurosurg. (2018) 13:11349. doi: 10.4103/ajns.AJNS_124_18

33. Sim Y-W, Min K-S, Lee M-S, Kim Y-G, Kim D-H. Recent changes in risk factors of chronic subdural hematoma. J Korean Neurosurg Soc. (2012) 52:234-9. doi: 10.3340/jkns.2012.52.3.234
34. Sousa EB, Brandão LF, Tavares CB, Borges IB, Neto NG, Kessler IM Epidemiological characteristics of 778 patients who underwent surgical drainage of chronic subdural hematomas in Brasília, Brazil. BMC Surg. (2013) 13:5. doi: 10.1186/1471-2482-13-5

35. Frati A, Salvati M, Mainiero F, Ippoliti F, Rocchi G, Raco A, et al. Inflammation markers and risk factors for recurrence in 35 patients with a posttraumatic chronic subdural hematoma: a prospective study. J Neurosurg. (2004) 100:2432. doi: $10.3171 /$ jns.2004.100.1.0024

36. Kolias AG, Chari A, Santarius T, Hutchinson PJ. Chronic subdural haematoma: modern management and emerging therapies. Nat Rev Neurol. (2014) 10:570-8. doi: 10.1038/nrneurol.2014.163

37. Yang AI, Balser DS, Mikheev A, Offen S, Huang JH, Babb J, et al. Cerebral atrophy is associated with development of chronic subdural haematoma. Brain injury. (2012) 26:1731-6. doi: 10.3109/02699052.2012.698364

38. Moon K-S, Lee J-K, Kim T-S, Jung S, Kim J-H, Kim S-H, et al Contralateral acute subdural hematoma occurring after removal of calcified chronic subdural hematoma. J Clin Neurosci. (2007) 14:283-6. doi: 10.1016/j.jocn.2005.11.016

39. Wang H, Wang C, Li Z. Recurrent bilateral chronic subdural hematoma after interventional embolization combined with drilling and drainage treatment. $J$ Craniofac Surg. (2020) 31:e171-3. doi: 10.1097/SCS.0000000000006128

40. Sirh S, Park HR, Park SQ. Usefulness of middle meningeal embolization to prevent recurrent spontaneous chronic subdural hemorrhage. J Cerebrovasc Endovasc Neurosurg. (2018) 20:40-6. doi: 10.7461/jcen.2018.20.1.40

41. Fan G, Wang H, Ding J, Xu C, Liu Y, Wang C, et al. Application of absolute alcohol in the treatment of traumatic intracranial hemorrhage via interventional embolization of middle meningeal artery. Front Neurol. (2020) 11:824. doi: 10.3389/fneur.2020.00824

Conflict of Interest: The authors declare that the research was conducted in the absence of any commercial or financial relationships that could be construed as a potential conflict of interest.

Publisher's Note: All claims expressed in this article are solely those of the authors and do not necessarily represent those of their affiliated organizations, or those of the publisher, the editors and the reviewers. Any product that may be evaluated in this article, or claim that may be made by its manufacturer, is not guaranteed or endorsed by the publisher.

Copyright $\odot 2021$ Wei, Fan, Li, Wang, Li, Wang and Li. This is an open-access article distributed under the terms of the Creative Commons Attribution License (CC $B Y)$. The use, distribution or reproduction in other forums is permitted, provided the original author(s) and the copyright owner(s) are credited and that the original publication in this journal is cited, in accordance with accepted academic practice. No use, distribution or reproduction is permitted which does not comply with these terms. 